\title{
Zu Risiken und Nebenwirkungen ...? Anregung zur Diskussion über potenzielle Nebenwirkungen klinisch-psychologischer Diagnostik in der Psychotherapie
}

\author{
Kevin Hilbert Andrea Ertle \\ Institut für Psychologie, Humboldt-Universität zu Berlin, Berlin, Deutschland
}

\section{Schlüsselwörter}

Diagnostik · Strukturiertes Interview · Aufklärung und

Einwilligung · Nebenwirkungen · Qualitätssicherung

\section{Zusammenfassung \\ Der Wert einer umfassenden, strukturierten Diagnostik für Therapieplanung und -durchführung ist unumstritten. Über mögliche Nebenwirkungen und Risiken wird aber selten dis- kutiert oder aufgeklärt. Ebenso steht die Bedeutung der Aufklärung von Patient:innen über die Psychotherapie außer Frage, während die Bedeutung der Diagnostik in der Auf- klärungsroutine weniger Raum einnimmt. Unter Heran- ziehen von zwei Fallbeispielen werden mögliche Neben- wirkungen eines umfangreichen diagnostischen Vorgehens beleuchtet, darunter soziale Konsequenzen, (Selbst-)Stig- matisierung und die Bedeutung von Lebenszeitdiagnosen. Eine bessere Aufklärung sowie gegebenenfalls eine Fokus- sierung angewandter diagnostischer Instrumente werden als Veränderungen des diagnostischen Prozesses vorge- schlagen. Gerne möchten wir eine weitere Diskussion zum Thema anregen. \\ (c) 2021 S. Karger AG, Basel}

\section{On Risks and Side Effects: Encouraging a Discussion on Potential Side Effects of the Diagnostic Process in Psychotherapy}

\section{Keywords}

Diagnostics · Structured clinical interview · Informed consent $\cdot$ Side effects $\cdot$ Quality control

\begin{abstract}
A broad and structured diagnostic approach is highly valuable for treatment conception and implementation. However, potential side effects and risks are rarely subject to discussion or informed consent. While the importance of informed consent for patients of psychotherapy is apparent, the relevance of including diagnostic procedures in patient information seems less clear. Using two case descriptions, we discuss the potential side effects of a broad diagnostic approach, including social consequences, self-stigma, and the significance of lifetime diagnoses. Options for adapting the diagnostic process include a more comprehensive patient information and, optionally, a more focused use of diagnostic tools. We would be very happy to encourage a more in-depth discussion on the topic.

(c) 2021 S. Karger AG, Basel
\end{abstract}

\section{Einleitung}

Eine umfassende, strukturierte Diagnostik ist für die psychotherapeutische Praxis äußerst wertvoll: Sie ermöglicht eine Indikationsstellung und passgenaue Therapieplanung, minimiert diagnostische Fehler und erfüllt in Verbindung mit der Psychoedukation der Patient:innen sogar eine therapeutische Funktion [Hoyer \& Knappe, 2012]. Insbesondere in Forschungs- und Ausbildungsambulanzen sind dabei eine Reihe von strukturierten bzw. standardisierten Interviews im Einsatz, deren gute Reliabilität und Validität als belegt gelten darf [DIPS: Margraf et al., 2017; DIA-X-5: Hoyer et al., 2020; SCID- 
5-CV: Osório et al., 2019]. Ein zentraler Vorteil der Benutzung dieser Interviews wird darin gesehen, dass diese nicht nur die Erhebung und Bewertung diagnostischer Information unterstützen, sondern gleichzeitig so umfangreich sind, dass das Risiko übersehener Diagnosen gering ist [Hoyer \& Knappe, 2012]. Die Autor:innen skizzieren das treffende Beispiel einer markanten depressiven Erkrankung, deren Wiederauftreten allerdings durch eine weniger offensichtliche, gleichzeitig bestehende Angststörung ausgelöst wird. Letztere könnte bei einem weniger strukturierten Vorgehen nicht erkannt werden. Umgekehrt wird für besonders imponierende Syndrome weniger schnell eine Diagnose vergeben, sondern es werden trotz augenscheinlicher Plausibilität alle diagnostischen Kriterien geprüft. Auch Gedächtnisfehler, beispielsweise hinsichtlich einzelner diagnostischer Kriterien, wirken sich weniger stark aus. In der Summe führen diese Vorteile zu einer Reduktion falsch-positiver und falsch-negativer Urteile. Die strukturierte Diagnostik weist dabei eine vergleichsweise gute Reliabilität und $\mathrm{Va}$ lidität auf [beispielhaft für den CIDI: Andrews und Peters, 1998].

Die vielfältigen Vorteile strukturierter Diagnostik sind also klar und anerkannt. Gleichzeitig sehen wir bei diesem Vorgehen jedoch mögliche Nebenwirkungen und Risiken, die bisher weniger betrachtet und in der Praxis berücksichtigt werden. Im Folgenden möchten wir dies durch zwei fiktive, aber durchaus realistische Fallbeispiele illustrieren, einige wesentliche Aspekte genauer beleuchten und potenzielle negative Folgen skizzieren. Insgesamt ist es unser Anliegen, zu einer Diskussion des diagnostischen Vorgehens einzuladen und eine mögliche Erweiterung der Aufklärungspflicht auf diesen Teil einer Behandlung anzuregen. Wir stellen die strukturierte Diagnostik, die wir Autor:innen selbst in unserer klinischen Praxis nutzen, nicht infrage, sondern möchten an ihrer Verbesserung und verantwortungsvollen Implementierung mitwirken.

\section{Fallbeispiel 1}

Frau D., 25 Jahre alt, wendet sich an die Ambulanz für Psychotherapie, wo ein strukturiertes diagnostisches Interview durchgeführt wird. Sie leide seit einem knappen Jahr unter Angstanfällen, einhergehend mit starken körperlichen Symptomen (Herzrasen, Zittern, etc.) und der Angst zu sterben, die sie zu Beginn wie aus heiterem Himmel erwischt hätten. Zweimal habe sie deshalb bereits den Notruf gerufen. Sämtliche kardiologischen und weiteren Untersuchungen seien ohne Befund geblieben. Aus Angst vor weiteren Angstanfällen habe sie ihren Bewegungsradius inzwischen stark eingeschränkt. Auf Nachfrage berichtet sie, nach der Trennung ihrer Eltern im Alter von
16 Jahren zu Hause aus- und in eine WG gezogen zu sein. Sie habe damals ein Jahr lang täglich Alkohol getrunken, "gekifft" und wiederholt "die Schule geschwänzt". Dann habe sie sich aber wieder gefangen und die Schulzeit mit dem Abitur abgeschlossen. Nach einem freiwilligen sozialen Jahr studiere sie nun und lebe mit ihrem Freund in einer kleinen Wohnung.

Mit der Hoffnung, dass man ihr bezüglich der Ängste und der daraus resultierenden Einschränkungen helfen könne, sei sie in die Ambulanz gekommen. Nun wisse sie nicht, wie sie die rückgemeldeten Diagnosen einordnen solle: Agoraphobie mit Panikstörung sowie Alkoholabhängigkeit (gegenwärtig abstinent). Sie sei einerseits froh, für die Angstzustände einen Namen zu haben. Als Alkoholabhängige die Ambulanz zu verlassen, irritiere sie aber. Bis eben hatte sie gedacht, die Zeit damals hinter sich gelassen zu haben; nun komme sie sich kränker vor als gedacht.

\section{Fallbeispiel 2}

Der achtjährige C. kommt mit seinen Eltern in die psychotherapeutische Ambulanz für Kinder und Jugendliche. Seine Angst vor Hunden sei so ausgeprägt, dass er den Weg zur Schule nicht mehr alleine zurücklegen möchte und er seine Freundin nicht besuchen könne, da deren Familie einen Hund habe. Bei gemeinsamen Spaziergängen wolle er am liebsten nicht mitgehen, und wenn ihnen ein Hund begegne, beginne er zu weinen. Neben dem diagnostischen Interview wird routinemäßig ein Intelligenztest durchgeführt. C. und seine Eltern verlassen die Ambulanz mit folgenden Informationen: Diagnose spezifische Phobie und normale Intelligenz (IQ von 94). Die Hoffnung auf eine Linderung der Ängste ihres Sohnes ist begleitet von Fragen zur Bedeutung der Intelligenzeinschätzung, die zuvor nie ein Thema gewesen war. In der Folge kommt es in der Familie zu Verunsicherung, erhöhter (Selbst-)Aufmerksamkeit für Schulleistungen und, in dem Glauben, unterdurchschnittlich begabt zu sein, zu einer negativen selbsterfüllenden Prophezeiung.

Beiden Fallbeispielen ist gemein, dass die Patient:innen zum einen zumindest von Teilen der rückgemeldeten Information überrascht sind, insofern, dass sie gar nicht davon ausgingen, dass dieser Bereich Teil der Diagnostik sein würde, und die Bedeutung dieser Rückmeldung ebenso nur schwer einschätzen können. Zum anderen haben beide Rückmeldungen zumindest unmittelbar negative Folgen für die Patient:innen, beispielsweise durch entsprechende Sorgen, insbesondere im ersteren Fall sind auch langfristige negative Konsequenzen möglich. Beide Aspekte möchten wir im Folgenden gerne vertiefen. 


\section{Besteht ein Mandat für eine umfangreiche Diagnostik über eine Vielzahl verschiedener Störungsgruppen?}

Patient:innen kommen in den meisten Fällen mit einem Auftrag, einem Anliegen oder zumindest einem mehr oder minder diffusen Problem in die Behandlung. Insbesondere wenn daraufhin eine breite klassifikatorische Diagnostik erfolgt, können jedoch über das geäußerte Anliegen hinaus noch weitere klinisch relevante Beschwerden und Störungen sichtbar werden. Dies ist insbesondere bei der Verwendung umfangreicher strukturierter oder standardisierter klinischer Interviews der Fall. Es stellt sich jedoch die Frage, ob für eine umfassende Diagnostik und darüber hinaus eine Weitergabe des Ergebnisses (z. B. an Kostenträger:innen) grundsätzlich ein Mandat besteht.

Ein Vergleich mit der somatischen Medizin mag polemisch erscheinen, erlaubt aber vielleicht einen Perspektivwechsel: sucht man im Winter mit Fieber und Gliederschmerzen eine ärztliche Praxis auf, wird die initiale Diagnostik sich in den meisten Fällen auf eine erste Abklärung der relevanten Infektionskrankheiten beschränken, also beispielsweise grippale Infekte, Influenza und in den aktuellen Zeiten das Coronavirus. Dies mag zum Beispiel ein Blutbild und damit auch die Möglichkeit von Zufallsbefunden einschließen, die Diagnostik würde sich aber initial auf die vorgetragenen Beschwerden beschränken. Eine Abklärung verschiedenster Krebserkrankungen oder - um ein gesellschaftliches Stigma hinzuzufügen - einschlägiger Geschlechtskrankheiten fände zu diesem Zeitpunkt eher nicht statt. Der Vergleich zur somatischen Medizin lässt es vielleicht weniger zwangsläufig erscheinen, mit welcher Breite das diagnostische Vorgehen auf unserem Gebiet angelegt ist. Zudem dürften die Erwartungen bei Haus- und Fachärzt:innen die Erwartungen unserer Patient:innen entscheidend prägen, insbesondere wenn es sich um die erste Psychotherapie handelt.

Nach unserem Verständnis wird die erforderliche sowie angemessene Diagnostik durch das Spannungsfeld zwischen dem Auftrag der Patient:innen und den Erfordernissen für eine Behandlung lege artis bestimmt. Dabei schließt das Selbstbestimmungsrecht der Patient:innen in der Regel die Möglichkeit ein, sich mit bestimmten Beschwerden nicht oder zumindest nicht zu diesem Zeitpunkt zu befassen. Patient:innen haben neben dem Recht auf Selbstbestimmung auch ein Recht auf Nicht-Wissen [Klinkhammer, 2012]; Therapeut:innen unterliegen hingegen einer Fürsorgepflicht [Heidenreich, 2020], gleichzeitig gilt aber das Gebot der Nichtschädigung [Beauchamp und Childress, 2019].

Insofern scheint die Annahme fraglich, dass $\mathrm{Pa}$ tient:innen, die sich mit einem bestimmten Anliegen vor- stellen, gleichzeitig eine Diagnostik oder Behandlung aller abklärbaren Beschwerden über die Lebensspanne wünschen. Natürlich erleben viele Patient:innen ihre Symptome erst einmal recht diffus, und häufig werden sich störungsübergreifende Zusammenhänge zwischen einzelnen Beschwerden zeigen. Dies begründet jedoch nicht unbedingt einen Automatismus zur möglichst breiten Diagnostik. Vielmehr werden zusätzliche diagnostische Maßnahmen vor allem durch die Abklärung von Indikation bzw. Kontraindikation therapeutischer Maßnahmen begründet. Diese jedoch gehen ihrerseits auf den Patient:innenauftrag zurück. Es dürfte schwierig sein, diagnostische Maßnahmen mit einer möglichen Kontraindikation für eine Intervention zu rechtfertigen, die die Patient:innen gar nicht wünschen. Für Patient:innen, die eine Behandlung von Panikattacken in öffentlichen Verkehrsmitteln wünschen, ist eine umfangreiche Angstdiagnostik sowie eine Abklärung möglicher Kontraindikationen für eine Expositionsbehandlung also angemessen, während beispielsweise eine frühzeitige Diagnostik zu möglicherweise komorbiden Essstörungen weniger klar begründbar scheint. Dies spräche für eine Diagnostik, die einerseits zielgerichtet ist, also eine spezifische diagnostische Fragestellung beantworten will, und die sich andererseits an den Grundsätzen der Datensparsamkeit orientiert. Eine solche Diagnostik würde dann nur diejenigen Instrumente enthalten, die zur Beantwortung der diagnostischen Fragestellung zwingend erforderlich sind, und bedarf auf dieser Grundlage der Einwilligung der Patient:innen.

Mitunter scheint eine besonders umfangreiche Diagnostik auch im Wunsch nach einer umfassenden Charakterisierung der Patient:innenstichprobe für die Forschung oder mit dem vertieften Erwerb diagnostischer Kompetenzen von in Ausbildung befindlichen Psychotherapeut:innen begründet zu liegen. Hier gestaltet sich der diagnostische Auftrag aus unserer Perspektive jedoch weniger einschlägig, da er nicht unter die Absicht einer individuellen Heilbehandlung lege artis subsumiert werden kann. In einem für die Patient:innen optionalen, mit zusätzlicher Aufklärung und Einwilligung verbundenen Bestandteil der Diagnostik könnte dieses Ziel verfolgt werden, nicht jedoch in der obligatorischen Standarddiagnostik.

\section{Die Rolle der Patient:innenaufklärung}

Die Rolle der Aufklärung von Personen, die Psychotherapie aufsuchen, ist unumstritten - so könnte man meinen. Tatsächlich scheint dies jedoch hauptsächlich für die eigentliche Behandlung im Sinne konkreter Interventionen zu gelten. Hier ist klar, dass eine Aufklärung über die beabsichtigte Intervention in Übereinstimmung 
mit den einschlägigen Gesetzen, Richtlinien und Verordnungen im Vorhinein zu erfolgen hat und dass diese Aufklärung insbesondere mögliche Risiken sowie gegebenenfalls Alternativen benennt. Weniger klar scheint indes zu sein, dass (ob?) dies ebenso für die Diagnostik gilt.

Die Muster-Berufsordnung der Bundespsychotherapeutenkammer beispielsweise spricht im $\$ 7$ “Aufklärung" explizit nur die "psychotherapeutische Behandlung" an [Bundespsychotherapeutenkammer, 2018, S. $10]$. Ebenso halten es $\$ 630$ c des BGB nach der Änderung durch das Gesetz zur Verbesserung der Rechte von Patientinnen und Patienten (vulgo Patientenrechtegesetz) sowie die Berufsethische Richtlinie der Deutschen Gesellschaft für Psychologie e.V. [Deutsche Gesellschaft für Psychologie, 2016, S. 31]. Möglicherweise wird die geschilderte Wortwahl mitunter als uneindeutig empfunden oder sogar als tatsächlich nur die eigentlichen Interventionen betreffend verstanden. Dies halten wir jedoch für unzureichend. Der Zweck der obigen Regelungen besteht im Schutz der Patient:innen vor möglicherweise schädigenden Eingriffen und in der Bewahrung ihrer Autonomie [cf. Beauchamp und Childress, 2019]. Aufgrund möglicher negativer Folgen einer Diagnosestellung stellt die Diagnostik hier selbst einen Eingriff dar.

Weiterhin mag der Vergleich mit entsprechenden Regelungen der ärztlichen Kolleg:innen lohnen: Die (Muster-)Berufsordnung für die in Deutschland tätigen Ärztinnen und Ärzte spricht hier im relevanten $\$ 8$ "Aufklärungspflicht” zwar ebenfalls allgemein davon, dass die Einwilligung zur "Behandlung" notwendig sei, notiert aber später, dass "vor diagnostischen oder operativen Eingriffen" [Bundesärztekammer, 2018, S. 4] eine Bedenkzeit zu gewährleisten sei. Kommentare zur Patient:innenaufklärung im Deutschen Ärzteblatt spezifizieren zudem explizit die Notwendigkeit einer Aufklärung und Einwilligung auch vor diagnostischen Prozeduren [Parzeller et al., 2007; Bundesärztekammer, 1990]. Die Schlussfolgerung aus diesen Überlegungen ist also, dass auch die initiale Diagnostik bereits einer angemessenen Aufklärung über möglicherweise negative Folgen einer bloßen Diagnosestellung sowie einer expliziten Einwilligung der Patient:innen bedarf. Dies gilt umso mehr, wenn diese breit angelegt ist und möglicherweise über den ausdrücklichen Auftrag der Patient:innen hinausgeht.

\section{Konsequenzen der aktuellen Praxis}

\section{Soziale Konsequenzen}

Die aktuelle Praxis einer breit angelegten Diagnostik bei gleichzeitig schmaler Aufklärung birgt die Gefahr einer Reihe negativer Konsequenzen. So können sich Patient:innen unvorbereitet mit Diagnosen konfrontiert sehen, die beispielsweise das Ergreifen bestimmter beruflicher Laufbahnen, das Abschließen von Berufsunfähigkeits- oder privater Krankenversicherungen oder eine Verbeamtung erschweren. Es ist nicht auszuschließen, dass eine solche Entwicklung zu einer weiteren Verschärfung der psychischen Belastung beitragen kann, beispielsweise durch neu oder verstärkt auftretende Sorgen, Niedergeschlagenheit, Infragestellen der eigenen Person usw., insbesondere bei einer ohnehin fragilen psychischen Verfassung, die Anlass für die Untersuchung ist. Anstelle von Unterstützung erführen die Patient:innen zunächst möglicherweise eine Mehrbelastung - und dies ohne angemessene vorherige Aufklärung.

Wenn umgekehrt über die Risiken einer ausführlichen, breiten Diagnostik aufgeklärt wird, besteht allerdings die Gefahr, dass Patient:innen zu übertriebener Vorsicht verleitet werden, sie bestimmte Beschwerden, dysfunktionale Verhaltensweisen oder Gegebenheiten in ihrem Bericht aus Sorge um negative Konsequenzen aussparen oder kleinreden. Dies könnte selbst dann der Fall sein, wenn eine ausführliche Diagnostik gar keine problematischen Diagnosen zutage fördert, und den meisten Patient:innen fällt es sicherlich schwer, das Risiko der Preisgabe einzelner Informationen im Vorfeld korrekt abzuschätzen.

\section{Angemessenheit von Lebenszeitdiagnosen}

Neben einer breiten Erfassung der aktuellen Beschwerden von Patient:innen ist es auch üblich, retrospektiv über die Lebensspanne Symptome und Syndrome zu erfragen. Für verschiedene Diagnosen, z. B. Schizophrenie, affektive Störungen oder Störungen im Zusammenhang mit psychotropen Substanzen, wird nach ICD oder DSM stets auch die aktuelle relative Ausprägung (z. B. leicht-, mittel-, schwergradig bzw. teil- oder vollremittiert) kodiert. Ohne das Konzept der Lebenszeitdiagnosen an sich an dieser Stelle vertieft diskutieren zu wollen, stellt sich zumindest die Frage, ob es sinnvoll und ethisch ist, wie in Fallbeispiel 1 eine aktuell remittierte Lebenszeitdiagnose retrospektiv erstmalig aktenkundig zu vergeben, wenn dieser Vorgang keinerlei Vorteile, aber potenziell gravierende Nachteile für die Patient:innen hat.

\section{(Selbst-)Stigmatisierung und Hilfesuchverhalten}

Darüber hinaus gibt es weitere mögliche Nebenwirkungen einer Diagnose, die man trotz remittierter Symptomatik nicht wieder ablegen kann, insbesondere im Hinblick darauf, ob dies Personen mit den genannten Diagnosen dazu animiert, Hilfsangebote aufzusuchen. So vertreten Bühringer und Rumpf [2018] die These, dass Stigmatisierung, verzögerter Behandlungsbeginn und die geringe Erreichbarkeit von Menschen mit einer Suchterkrankung zusammenhängen. In ihrer S3-Leitlinie Schizophrenie weist die DGPPN [2019] darauf hin, dass Stig- 
ma und Diskriminierung sogar als eine zur eigentlichen Diagnose zusätzlich zu bewältigende Erkrankung betrachtet werden kann, die sie als "Stigma-Coping" (S. 21) bezeichnen. Eine Zusammenschau empirischer Studien zu diesem Thema beschreibt die Folgen von (Selbst-)Stigmatisierung als "Why-try-Effekt": (Selbst-)Stigmatisierung kann, vermittelt über geringen Selbstwert und reduzierte Selbstwirksamkeit, schließlich dazu führen, dass Betroffene davon abgehalten werden, professionelle Hilfe in Anspruch zu nehmen [Corrigan et al., 2009].

\section{Fazit und Einladung zur Diskussion}

Die Vorteile einer umfassenden, strukturierten klinischen Diagnostik sind gut belegt. Gleichzeitig gibt es auch einzelne negative Aspekte dieses Vorgehens, die möglicherweise weniger präsent sind und entsprechend weniger Berücksichtigung finden. Im vorliegenden Diskussionspapier sollen mögliche Nachteile für Patient:innen genauer beleuchtet und das Bild der strukturierten Diagnostik entsprechend ergänzt werden.

Aus unserer Perspektive gibt es mindestens zwei Optionen, diese Nachteile in Zukunft zu mindern oder zu adressieren. Dabei beziehen wir uns ausschließlich auf Optionen, die wir als Kliniker:innen in der Hand haben - langfristige Bemühungen beispielsweise zum Abbau von Stigmatisierungen oder sozialrechtlichen Nachteilen lassen wir daher bewusst außen vor, obwohl wir diese selbstverständlich begrüßen. Die erste Möglichkeit besteht in der Ergänzung der Patient:innenaufklärung spezifisch um mögliche Risiken und negativen Folgen des diagnostischen Prozesses. Diese sollte beispielsweise den Hinweis enthalten, dass alleine die aktenkundige Feststellung bestimmter Diagnosen möglicherweise zu jahreoder lebenslangen Nachteilen führen kann. Etwas weiter gedacht könnten kritische Aspekte des diagnostischen Vorgehens, wie beispielsweise die Leistungsdiagnostik des Kindes in Beispiel 2, zusammen mit den Patient:innen und gegebenenfalls Sorgeberechtigten erarbeitet werden, um ein besseres Verständnis zu erzielen, was mit welchen Folgen wie erhoben wird. Dieses Vorgehen wäre sicherlich je nach Umfang relativ gut praktikabel und würde keine grundlegende Veränderung des aktuellen diagnostischen Vorgehens erfordern. Allerdings könnte dies zu einer Verunsicherung der Patient:innen führen und letztlich damit einhergehen, dass sie insgesamt weniger offen berichten. Zudem wäre weiterhin zumindest offen, ob das diagnostische Vorgehenimmer vom Patient:innenauftrag abgedeckt ist.

Eine zweite Möglichkeit läge in einer weniger breit angelegten Diagnostik, die sich mehr auf diejenigen Störungsbereiche einschränkt, die von den Patient:innen selber ins Spiel gebracht werden. Weitere diagnostische In- formationen würden dann verstärkt im Therapieverlauf nach Bedarf und Anlass eingeholt. Dieses Vorgehen würde sich enger am Auftrag der Patient:innen orientieren, allerdings eine gewisse Abkehr vom bisher üblichen diagnostischen Prozess bedeuten und möglicherweise ein erhöhtes Risiko bergen, relevante Informationen gar nicht oder erst später im Therapieprozess zu erheben.

Sowohl das bisherige Standardprozedere als auch die beiden vorgeschlagenen Alternativen gehen jeweils mit Vor- und Nachteilen einher. Sicherlich gibt es weitere Möglichkeiten, an die wir bei unseren ersten dargelegten Überlegungen nicht gedacht haben. Die Perspektive unserer Kolleg:innen ist daher von großem Interesse für uns. Wir würden uns freuen, zumindest eine Reflexion möglicher Nachteile des strukturierten diagnostischen Prozesses für unsere Patient:innen angeregt zu haben, die im Idealfall einen Beitrag zur Reduktion dieser Nachteile leistet.

\section{Acknowledgment}

Die Autor:innen bedanken sich bei Ulrike Lüken, Eva Kischkel sowie Björn Elsner für die hilfreichen Kommentare und Anregungen.

\section{Statement of Ethics}

Die Autor:innen bestätigen hiermit, dass für die vorliegende Arbeit kein Ethikvotum erforderlich ist, da weder menschliche noch tierische Teilnehmer:innen oder Materialien zur Ausführung der Studie benötigt wurden. Die beiden Fallbeispiele sind fiktiv.

\section{Conflict of Interest Statement}

Die Autor:innen erklären, dass kein Interessenkonflikt besteht.

\section{Funding Sources}

Die Studie bzw. Veröffentlichung wurden nicht finanziell unterstützt.

\section{Author Contributions}

A. Ertle und K. Hilbert haben alle wesentlichen Schritte dieser Arbeit gemeinschaftlich unternommen, darunter die Entwicklung des Konzepts, die Erarbeitung eines ersten Textentwurfs sowie die kritische Überarbeitung. 


\section{Literatur}

Andrews G, Peters L. The psychometric properties of the Composite International Diagnostic Interview. Soc Psychiatry Psychiatr Epidemiol. 1998 Feb;33(2):80-8.

Beauchamp TL, Childress JF. Principles of biomedical ethics. 8th ed. New York: Oxford University Press; 2019.

Bühringer G, Rumpf HJ. Future of addiction treatment: plea for a paradigmatic change [Zukunft der Suchtkrankenversorgung: Plädoyer für einen Paradigma-Wechsel]. Sucht. 2018;64(3):125-8.

Bundesärztekammer. Empfehlungen zur Patientenaufklärung.Dtsch Arztebl. 1990;87(16):A127983.

Bundesärztekammer. (Muster-)Berufsordnung für die in Deutschland tätigen Ärztinnen und Ärzte - MBO-Ä 1997-*). In der Fassung der Beschlüsse des 121. Deutschen Ärztetages 2018 in Erfurt geändert durch Beschluss des Vorstandes der Bundesärztekammer am 14.12.2018. 2018. Verfügbar unter: https://www.bundesaerztekammer.de/fileadmin/user_upload/downloads/pdf-Ordner/MBO/MBO-AE.pdf.

Bundespsychotherapeutenkammer. MusterBerufsordnung für die psychologischen Psychotherapeutinnen und Psychotherapeuten und Kinder- und Jugendlichenpsychothera- peutinnen und Kinder- und Jugendlichenpsychotherapeuten. In der Fassung des Beschlusses des 24. Deutschen Psychotherapeutentages in Berlin am 17. Mai 2014, geändert mit dem Beschluss des 33. Deutschen Psychotherapeutentages in Berlin am 17. November 2018. Verfügbar unter: https://www.bptk.de/ wp-content/uploads/2019/01/Muster-Berufsordnung_der_BPtK.pdf.

Corrigan PW, Larson JE, Rüsch N. Self-stigma and the "why try" effect: impact on life goals and evidence-based practices. World Psychiatry. 2009 Jun;8(2):75-81.

Deutsche Gesellschaft für Psychologie. Berufsethische Richtlinien des Berufsverbandes Deutscher Psychologinnen und Psychologen e.V. und der Deutschen Gesellschaft für Psychologie e.V. 2016. Verfügbar unter: https:// www.dgps.de/fileadmin/documents/Empfehlungen/ber-foederation-2016.pdf.

DGPPN. S3-Leitlinie Schizophrenie. Langfassung. 2019. Verfügbar unter: https://www. awmf.org/leitlinien/detail/ll/038-009.html.

Heidenreich KS. Medizinische Zufallsbefunde in der Diagnostik und Forschung. Eine rechtsethische Analyse im Spannungsfeld zwischen Selbstbestimmungsrecht und ärztlicher Fürsorge. Berlin: Peter Lang; 2020.
Hoyer J, Knappe S. Psychotherapie braucht strukturierte Diagnostik! PiD Psychother Dialog. 2012;13(1):2-5.

Hoyer J, Voss C, Strehle J, Venz J, Pieper L, Wittchen HU, et al. Test-retest reliability of the computer-assisted DIA-X-5 interview for mental disorders. BMC Psychiatry. 2020 Jun;20(1):280.

Klinkhammer G. Arbeitskreis medizinischer Ethik-Kommissionen: eine klare Vereinbarung treffen. Dtsch Arztebl. 2012;109:33-4.

Margraf J, Cwik JC, Pflug V, Schneider S. Strukturierte klinische Interviews zur Erfassung psychischer Störungen über die Lebensspanne: Gütekriterien und Weiterentwicklungen der DIPS-Verfahren. Z Klin Psychol Psychother. 2017;46(3):176-86.

Osório FL, Loureiro SR, Hallak JE, Machado-deSousa JP, Ushirohira JM, Baes CV, et al. Clinical validity and intrarater and test-retest reliability of the Structured Clinical Interview for DSM-5 - Clinician Version (SCID-5-CV). Psychiatry Clin Neurosci. 2019 Dec;73(12): 754-60.

Parzeller M, Wenk M, Zedler B, Rothschild M. Aufklärung und Einwilligung bei ärztlichen Eingriffen. Dtsch Arztebl. 2007;104(9):A57686. 